\title{
Management of oral focal infection in patients with asthmatic symptoms
}

\author{
Haryono Utomo \\ Dental Clinic \\ Faculty of Dentistry Airlangga University \\ Surabaya - Indonesia
}

\begin{abstract}
Asthma is commonly related to allergic diseases, nevertheless only $40 \%$ of asthma patients are related to allergy or atopy. There are some unknown etiologies of asthma that are still in researches. One of the possible causes of asthma is the multiple chemical sensitivity syndrome (MCS) which related to the "neurogenic switching hypothesis". Since rhinitis, sinusitis and asthma are closely related, treatments which are successfully reduce or eliminate the rhinitis and sinusitis symptoms should also be advantageous to asthma management. There were a lot of sinusitis treatments which reduced asthma symptoms such as nasal corticosteroid, diathermy and surgery. It was also been reported that oral focal infection might cause sinusitis. However, the involvement of oral focal infection in the etiopathogenesis of asthma was seldom discussed. The objective of this study is to propose a mechanism of the relationship between oral focal infection and asthma which is explained by the "neurogenic switching hypothesis". Two asthmatic patients who also had periodontal disease, pulpal and periapical infection were treated with conventional and/or surgical dental treatments. After the dental and periodontal treatments were completed, the usual triggers of severe asthma attacks such as cold and house dust did not elicit the asthma symptoms. It concluded that regarding to the disappearing of asthma symptoms, the elimination of oral focal infection had a beneficial effect in reducing asthma symptoms.
\end{abstract}

Key words: asthmatic symptom, oral focal infection management

Correspondence: Haryono Utomo, c/o: Fakultas Kedokteran Gigi Universitas Airlangga. Jln. Mayjend. Prof. Dr. Moestopo No. 47 Surabaya 60132, Indonesia. E-mail: dhoetomo@indo.net.id Telp. 031-5053195.

\section{INTRODUCTION}

Asthma is a chronic inflammatory disorder of the airways characterized by an obstruction of airflow, which may be completely or partially reversed with or without specific therapy. Airway inflammation is the result of interactions between various cells, cellular elements, and cytokines. In susceptible individuals, airway inflammation may cause recurrent or persistent bronchospasm, which causes symptoms including wheezing, breathlessness, chest tightness, and cough, particularly at night or after exercise. ${ }^{1}$

It is important to understand the role of atopic or allergy in asthma. Asthma and atopy may coexist, but not all asthmatics are atopic and only some atopic patients have asthma. Forty percent of asthmatics have atopy, the rest has an unknown etiology of asthma. Nevertheless, all asthmatics patients-regardless of the presence or absence of atopy-have the cardinal features that define asthma. ${ }^{2}$

Epidemiological studies have clearly shown that rhinitis and asthma are frequently concurrent. Therefore, the concept of "one airway-one disease" had been implicated in rhinitis and asthma management. Allergen avoidance, specific immunotherapy and intra nasal corticosteroid were able to reduce symptoms in allergic rhinitis and asthma. ${ }^{4-6}$
The neurogenic switching hypothesis was introduced by Meggs ${ }^{7}$ which meant to explain the etiopathogenesis of allergic disease (i.e. urticaria, food allergy, and asthma), migraine, arthritis and other inflammatory diseases. It proposed that a local inflammation was able to initiate another inflammation in other parts of the body. In addition, the neurogenic switching mechanism may also occur in the oral tissues. 8

According to Li et al. ${ }^{9}$ oral focal infections were able to elicit systemic symptoms by means of various mechanisms. Evidence-based case reports had been revealed that oral focal infection might induce sinusitis. However, the relationship between oral focal infection and asthma was rarely discussed.

The objective of this case report is to achieve a better knowledge about the relationship of oral focal infection and asthma based on the concept of neurogenic switching mechanism.

\section{CASE}

Case 1: A male, 43 years, who came to the Dental Clinic Faculty of Dentistry, Airlangga University. At that time he had a problem with the recurrent periapical abscess which 
elicited pain. An oral surgeon advised to have apical resection of the 16 .

He suffered from asthma and rhinitis symptoms for about 35 years. A lot of medications had been consumed. Asthma attack could be triggered by chemical odor (i.e. alcohol and perfume), fermented food or drink, humid and cold weather. In order to prevent serious asthma symptoms, he always bring metered-aerosol bronchodilator.

Extra orally, there was a slight asymmetry on the right cheek and a dull pain was felt upon palpation. Intra orally, the buccal fold of the 16 was somewhat raised, and palpation caused tenderness. There were a lot of restorations, including inlay in 46 and crowns in 16, 25, 35, 36.

Panoramic radiograph showed horizontal resorption on 1615,2526 . At the apex of 16 there was periapical abscess (Figure 1).

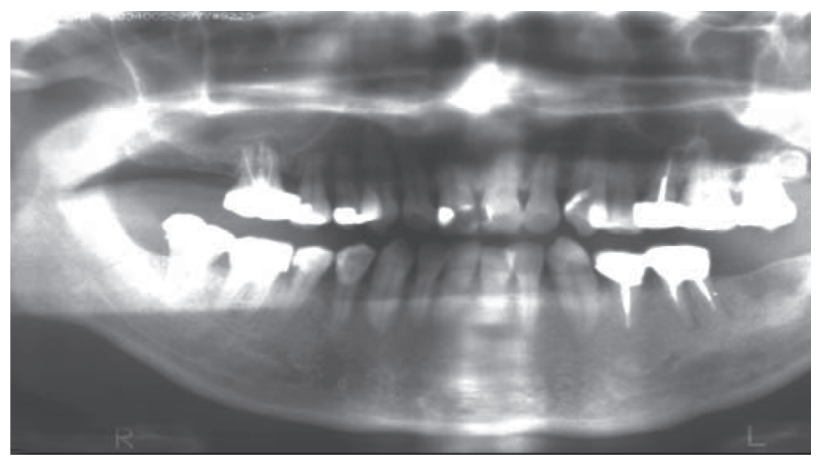

Figure 1. Panoramic radiograph pre apical resection of 16 .

Case 2: A male, 21 years who came to a private dental clinic. The chief complaint was pain on the upper left tooth, and also gingival bleeding. After the patient was told that oral infection might cause migraine, rhinitis and asthma symptoms, he told the dental practitioner about his suffering from rhinitis and asthma for about 10 years. A lot of medical treatment and alternative medicine had been tried to prevent asthma attack. The most recent prescription was aminophyllin tablets (oral bronchodilator) that had to be taken once daily.

Extra orally, the patient looked normal. Intra oral inspection showed that the patient suffered from generalized gingivitis and a deep caries in 24. The upper and lower gingival tissues were easily bled. Thermal testing on 24 revealed that the tooth remained vital, nevertheless it had increased sensitivity.

Panoramic radiograph (Figure 2) showed that alveolar resorption was absent. Apical dental radiograph indicated that perforation of the pulp chamber in 24 and periodontitis apicalis already occurred.

\section{CASE MANAGEMENT}

Case 1: Since the chief complaint was persistent pain and periapical abscess in 16 and endodontic treatment could not solve the problem, an oral surgeon decided to conduct an apical resection. The apical resection was done in the operation room of Oral Surgery Department, Faculty of Dentistry, Airlangga University. The patient was scheduled for the next visit in 5 days for removing the sutures. A few days subsequent to the surgical procedures, the abscess disappeared and also the rhinitis symptoms were reduced, followed by the asthma symptoms. Since then, the usual triggers of asthma such as chemical odors, fermented food, humid and cold weather did not elicit asthma symptoms.

At second visit, the surgical wound was healed and the sutures were removed. At that time the rhinitis symptoms (i.e. nasal congestion and rhinorrhea) that always started in the morning till the afternoon were diminished. Coincidentally, since the apical resection, the usual asthma symptoms also disappeared. The most recent evaluation was in June 2006, 3 years after the apical resection, the asthma symptoms did not reoccur.

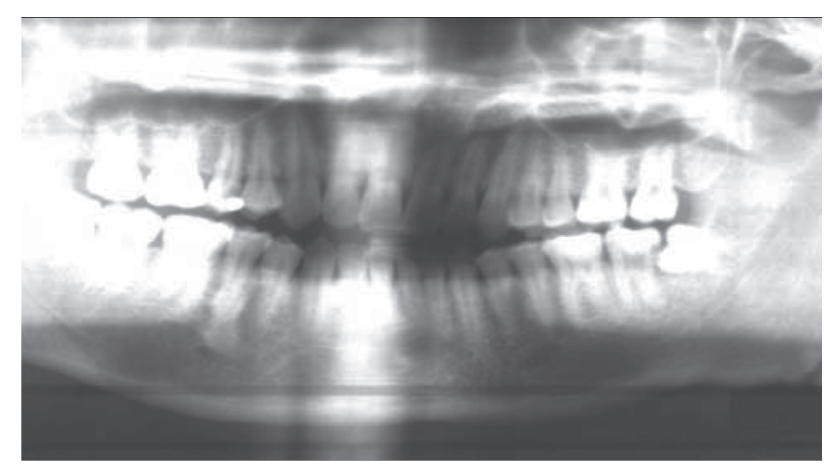

Figure 2. Panoramic radiograph pre-treatment.

Case 2: At the first visit, first attempt was the relief of pulpal pain followed by temporary restoration. Scaling had been done in every region which resulted in a lot of oozed dark red blood. Second visit was scheduled 4 days later. At the next visit the patient told that the asthma symptoms were disappearing, he only had a mild rhinitis in the morning; before that rhinitis could be happened all day long.

The pulpal condition was diagnosed as irreversible pulpitis. Root canal treatment was done by an endodontists which completed after 3 visits. Evaluations were done monthly for about one year after the last visit, the latest evaluation was in June 2006; the asthma symptoms did not reappear.

\section{DISCUSSION}

In the first case, there were two possible causes of oral focal infection which was the source of bacterial endotoxin lipopolysaccharides (LPS). The most possible cause was the periapical abscess in 16, although it had been treated endodontically, the periapical infection still in progress. The other cause was the chronic periodontitis which caused 
alveolar resorptions in 1615 and 25 26. The indication was that after apical resection, the rhinitis and asthmatic symptoms gradually disappeared.

Among all animals, humans are the most sensitive to the effects of endotoxins, which makes the knowledge of their biological effects on tissues fundamentally important. Endotoxins from vital or non vital, whole or fragmented bacteria act on macrophages, neutrophils and fibroblasts, ${ }^{10}$ and mast cells ${ }^{11}$ leading to the release of a large number of bioactive or cytokine chemical inflammatory mediators, such as tumor necrosis factor (TNF- $\alpha$ ), interleukin-1 (IL-1), IL-5, IL-8, alpha-interferon and prostaglandins. Furthermore, LPS is cytotoxic and acts as a potent stimulator of nitric oxide (NO) production. $9,10,12$

According to Leonardo et al., ${ }^{10}$ the bacterial endotoxin (LPS) adheres irreversibly to mineralized tissues, endodontic treatment and dressing only killed the bacteria. The inactivation of LPS by eliminating its biologically toxic potential that was the lipid A should be done with certain materials such as calcium hydroxide. Lipopolysaccharides and the products of degranulated mast cells were able to induced the neurogenic switching mechanism which then might elicit asthmatic symptoms.7,8,13 It seems plausible that the incomplete inactivation of LPS after endodontic treatment in the first case caused the ongoing periapical infection; the apical resection treatment then eradicate the remaining LPS.

In the second case, since it was diagnosed as pulpitis irreversible and the periapical inflammation was not severe; the major contribution of the LPS was suspected caused by the periodontal disease that was gingivitis. It was confirmed regarding to the immediate relief of asthma symptoms after scaling procedures whereas the endodontic treatment was not done yet.

In the periodontal disease, LPS originated from pathogenic gram negative bacteria such as porphyromonas gingivalis. Periodontal pockets act as cytokines and biofilms reservoir which may continuously release proinflammatory mediators that may cause systemic effects. The propagation of oral focal infection to systemic could be conducted by several mechanisms, via the blood stream ${ }^{9}$ or the neurogenic switching mechanism. ${ }^{7,8,13}$

The interplay between immunogenic and neurogenic inflammation was called the neurogenic switching mechanism. The neurogenic switching hypothesis that was proposed by Meggs, suggested that the etiopathogenesis of asthma was not merely related to atopy and immunogenic mechanism. The role of afferent nerve fibers stimulation which elicit neurogenic inflammation should also be considered. ${ }^{7}$

Meggs ${ }^{7}$ postulates that "neurogenic switching", or a crossover interaction of neurogenic and immunogenic inflammation, explains several puzzling aspects of inflammatory responses, including how an inflammatory stimulus applied to one tissue (e.g., skin) can result in inflammation in a different tissue (e.g., the lungs) and how neurogenic conditions such as migraine can be influenced by immunogenic agents. ${ }^{13}$

The interplay or cross-over of immunogenic and neurogenic inflammation which also termed the neurogenic switching mechanism was hypothesized to be involved in triggering asthmatic or other allergic symptoms. ${ }^{7,13}$ Neurogenic switching is proposed to result when a sensory impulse from a site of activation is rerouted via the central nervous system to a distant location to produce neurogenic inflammation at the second location. ${ }^{13}$

It also provides a mechanism to explain how allergens, infectious agents, irritants, and possibly emotional stress can exacerbate conditions such as migraine, asthma, and arthritis. The difference mechanism between atopic asthma that mostly related to immunogenic reaction and neurogenic mechanism of asthma are described in table $1 .^{7}$

According to Lundy and Linden, the neurogenic switching mechanism also occurred in the oral tissues. ${ }^{8}$ There were several factors which involved in neurogenic switching mechanism such as bacteria, toxins (lipopolysaccharides, LPS and proteoglycans, PGN), neuropeptides (substance P, SP and calcitonin gene-related peptide, CGRP) which able to degranulate mast cells. ${ }^{8,15}$ Afferent nerve fibers may in turn were stimulated by products of degranulated mast cells (i.e. histamine, tryptase) which then release neuropeptides. Other stimulators are the pro-inflammatory cytokines produced by LPS-induced macrophages, and bradykinin from damaged tissue. ${ }^{8}$

Case reports related to oral focal infection by Li et al. showed that sinusitis may be affected by oral infection. Since asthma and sinusitis were closely related, it seems plausible that oral focal infection may also involved in the etiopathogenesis of asthma, especially through the neurogenic switching mechanism. ${ }^{7-9,13,14}$ In these two cases,

Table 1. Immunogenic versus neurogenic asthma ${ }^{7}$

\begin{tabular}{lll}
\hline & Immunogenic & Neurogenic \\
\hline Triggered by & Protein aeroallergens & Volatile organic chemicals \\
Interacting with & IgE antibody & Chemical irritant receptors \\
Located on & Mast cells & Sensory nerve C-fibers \\
Releasing & Histamine, leukotrines, prostoglanolins, & Neuropeptides: substance P, neurokinen A, \\
& chemotactic factors & calcitonin gene-related peptide \\
Stimulating & Sensory nerve C-fibers & Mast cell degranulation \\
Producing & Bronchial inflammation & Bronchial inflammation \\
Manifesting as & Asthma & Asthma \\
\hline
\end{tabular}


the successful elimination of oral focal infection including the LPS was indicated by the reducing of asthma symptoms. The possible explanation of the diminished asthma symptoms was that after dental and periodontal treatments, there was an immediate cut off of the neurogenic switching mechanism which initiated by oral tissue inflammation.

In this case report, the patients also suffered from rhinitis which had a co-morbidity with asthma and may worsen asthma symptoms. Allergic rhinitis which suffered by $80 \%$ asthmatics has several sequelae and co-morbidities which impaired the quality of life that related to asthma, such as sinusitis, mouth breathing etc. (Figure 3)..$^{5}$ If the nose is obstructed, these individuals breathe with their mouth open, a practice that precipitates an asthma attack. Mouth breathers may resulting in dry mouth (xerostomia) which reduces the immune function of saliva as a part of mucosal immunity. 16

Regarding to the concept "one airway-one disease", which involved rhinitis, sinusitis and asthma. There are some explanation about the pathophysiologic relationship between sinusitis and asthma (Table 2), such as the aspiration of the mediators present in secretions. These mediators then reach the lower airway in inspired air, particularly at night, leading to deterioration in lung function, increased bronchial hyperreactivity, and symptoms on waking. $4,6,14,17$
The main mechanism whereby rhinitis and asthma are interrelated had been proposed as a systemic dissemination of mediators which are eosinophils, intercellular adhesion molecule-1 (ICAM-1) and vascular cell adhesion molecule-1 (VCAM-1) that were showed in nasal and bronchial mucosa after nasal provocation with allergen. Nasal provocation induced eosinophilia, leukocyte activation in peripheral blood, systemic propagation of inflammation from nasal to bronchial mucosa. ${ }^{6}$

Since the reducing of rhinitis and asthma symptoms in these patients happened in a short time without asthma medications, the possibility of neurogenic inflammation involvement was suspected. Regarding to the neurogenic inflammation in the orofacial region, the relationship between rhinosinusitis, asthma and the trigeminal nerve (CN V) had been studied. Experiments related to the nasobronchial reflex revealed that unilateral resection of the trigeminal nerve eliminated the bronchial resistance. The function of the trigeminal nerve is for the sense of irritation, also referred to chemestesis or the common chemical sense. 6,18

Further analysis of the interrelationship of the $\mathrm{CN} V$ and asthma was the existence of receptors in the nose and pharynx and, presumably, in the paranasal sinuses produce afferent fibers that form part of $\mathrm{CN} \mathrm{V}$. They passed to the brain stem and connects with the reticular formation of the

Allergic Rhinitis sequelae and co-morbidities

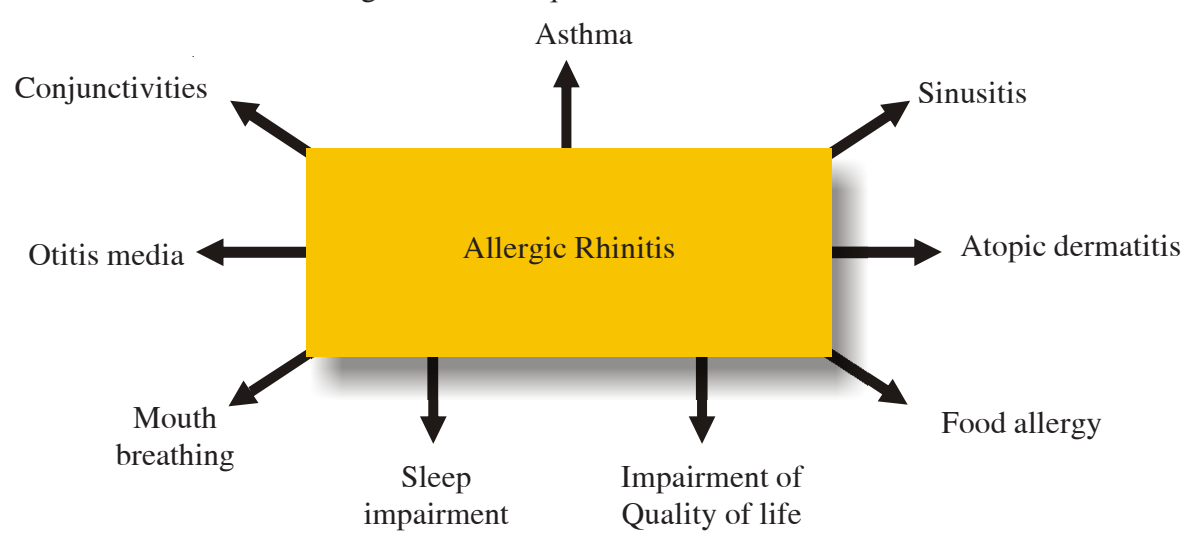

Figure 3. The sequelae and co-mordibidities of allergic rihinitis. ${ }^{5}$

Table 2. Proposed pathophysiologic mechanisms of asthma exacerbated by sinusitis ${ }^{14}$

$>$ Spread of inflammatory mediators and chemotactic factors to lower airways triggers sinobronchial reflex mechanism.

$>$ Stimulation of autonomic nervous system causes acute bronchial hyperresponsiveness.

$>$ Bronchoconstrictive reflexes originating in extrathoracic airway receptors are stimulated.

$>$ Reversible partial beta-adrenergic blockade is enhanced.

> Nasal congestion causes mouth-breathing, which leads to increased loss of water and heat in lower airways.

$>$ Depressed nitric oxide concentration promotes acute bronchial hyperresponsiveness.

$>$ Gastroesophageal reflux disease induces nasal mucosa edema, obstruction of sinus ostia, and stimulation of autonomic nervous system. 
dorsal vagal nucleus. From the vagal nucleus, parasympathetic efferent fibers travel in the vagus nerve to the bronchi. ${ }^{14}$

According to Doty, some free nerve endings of the $\mathrm{CN}$ $\mathrm{V}$ might terminate $1 \mu \mathrm{m}$ within the epithelial surface of the nose, such as in nasal vestibule and nasal chambers. They produce such sensations as irritation, tickling, burning, warming, cooling and stinging. In addition to serving chemosensory functions, $\mathrm{CN} \mathrm{V}$ fibers within the nasal vestibule mediate the tactile sensations of temperature and pressure. ${ }^{19}$

Trigeminal nerve stimulation can reflexively influence nasal engorgement, respiration rate, nasal secretion, and sneezing. Since most trigeminal stimulants were lipid soluble, such as volatile chemicals; the stimulations were likely. Stimulated CN V and the sphenopalatine ganglion (SPG) may referred to multiple chemical sensitivity syndrome (MCS) which initiated in the nasal cavity. $7,8,13,20,21$

The periapical and periodontal infection in both cases might be able to conduct the neurogenic switching mechanism, which may antidromically stimulate the sphenopalatine ganglion (SPG). The stimulation of SPG, a parasympathetic ganglion also proposed as an etiology of MCS, which related to rhinitis, allergy, asthma, migraine etc. Several medications which applied to the SPG were able to relief migraine, rhinitis and asthma symptoms. ${ }^{20}$ Since maxillary periodontal tissues are innervated by the sensory nerve fibers through the $\mathrm{CN}$ V2 and the parasympathetic nerve fibers through the SPG, there might be a correlation between periodontal disease and the SPG sensitization. ${ }^{8,22}$ Activation of the SPG by CN V2 which releases neuropeptides may cause the inflammation of the neighboring artery and mucosa. Inflamed nasal mucosa resulting in nasal congestion, sinusitis; and migraine. ${ }^{21}$

Regarding to the relief of the asthmatic symptoms, there was a case report regarding the possible relationship between periodontal inflammation, the SPG and sinusitis. As sinusitis is closely related to asthma, dental treatment which successfully treated sinusitis patient such as the "assisted drainage" method, was expected to give the same result to asthma patient. ${ }^{22,23}$ Periodontal treatment which conducted to the second case was similar to the "assisted drainage" method that was done due to assist drainage of pro-inflammatory mediators which may involve in the neurogenic switching mechanism in the periodontium. Immediate cut off of the mechanism was suggested to reduce the sinusitis and asthma symptoms. ${ }^{22}$

The stimulation of afferent nerve or parasympathetic fibers by pro-inflammatory mediators in periodontal diseases and pulpal inflammation may propagate to distant site through the neurogenic switching mechanism. It may indirectly affect bronchial hyperresponsiveness via the nasal inflammation which probably caused by the sensitized SPG. ${ }^{21,22}$ Sensitization of the SPG that might be caused by oral inflammation was able to induced nasal hypersensitivity. This condition increased the nasal susceptibility to provocations which stimulate the nasal sensory nerves that leads to sensations of pain and stuffiness.

This reaction was created by type $\mathrm{C}$ nociceptive nerve fibers which releases neuropeptides that increase plasma extravasation and glandular secretion. ${ }^{19,24}$ This axonal response acts as an immediate protective mucosal defense mechanism. Recruited parasympathetic reflexes cause submucosal gland secretion via acetylcholine and muscarinic M3 receptors. Itching, sneezing, and other avoidance behaviors rapidly clear the offending agents from the upper airways and protect the lower airways. ${ }^{24}$

Dysfunction of these nerves may contribute to allergic rhinitis, infectious rhinitis, nasal hyperresponsiveness, and possibly sinusitis. Sympathetic arterial vasoconstriction reduces mucosal blood flow, sinusoidal filling, and mucosal thickness, and so restores nasal patency. Loss of sympathetic tone may contribute to some chronic, nonallergic rhinopathies. ${ }^{22,24}$

Regarding to the relief of asthma symptoms after the completion of dental and periodontal treatments; it concluded that the elimination of oral focal infection is mostly probable in reducing asthma symptoms.

\section{REFERENCES}

1. Sharma G. Asthma. Available online at http://www.emedicine.com/ ped/pulmonology. Accessed May 15, 2006.

2. Terr AI. Asthma. In: Parlow TG, Stites DP, Terr AI, Imboden JP, editors. Medical immunology. 10 $0^{\text {th }}$ ed. Boston: McGraw-Hill; 2003. p. 359.

3. Ponsonby AL, Gatenby P, Glasgow N, Mullins R, McDonald T, Hurwitz M. Which clinical subgroups within the spectrum of child asthma are attributable to atopy. Chest 2002; 121:135-42.

4. Lipworth BJ, White PS. Allergic inflammation in the unified airway: start with the nose. Thorax 2000; 55:878-91.

5. Fokkens W, Bachert C. Comorbidity of rhinitis. The UCB Insitute of Allergy, 2004. p. 5-13.

6. Serrano C, Valero A, Picado C. Rhinitis and asthma: One airway, one disease. Arch Bronconeomol 2005; 41:569-78.

7. Meggs WJ. Neurogenic switching: a hypothesis for a mechanism for shifting the site of inflammation in allergy and chemical sensitivity. Env Health Perspect 1997; 105(S2):1-10.

8. Lundy W, Linden R. Neuropeptides and neurogenic mechanism in oral and periodontal inflammation. Crit Rev Oral Biol 2004; 15(2):82-98.

9. Li XJ, Kolltveit KM, Tronstad L, Olsen I. Systemic diseases caused by oral infection. Clin Microb Rev. 2000; 13(4):547-58.

10. Leonardo MR, da Silva RAB, Assed S, Nelson-Fiho P. Importance of bacterial endotoxin (LPS) in endodontics. J Appl Oral Sci 2004; 12(2):93-8.

11. Supajatura V, Ushio H, Nakao A, Akira S, Okumura K, Ra C, Ogawa H. Differential responses of mast cell Toll-like receptors 2 and 4 in allergy and innate immunity. J Clin Invest. 2002; 109:1351-9.

12. Madianos PN, Bobetsis YA, Kinane DF. Generation of inflammatory stimuli: how bacteria set up inflammatory responses in the gingiva. J Clin Periodontol 2005; 32(S6): 57-71.

13. Cady RK, Schreiber CP. Sinus headache or migraine. Neurology. 2002; 58:S10-S14.

14. Muller BA. Sinusitis and its relationship to asthma: can treating one airway disease ameliorate another? Postgrad medicine 2000; 108(5):55-61.

15. Walsh LJ. Mast cells and oral inflammation. Crit Rev Oral Biol Med 2003; 14(3):188-98. 
16. Laurikainen K. Asthma and oral health: A clinical and epidemiological study. Academic dissertation. Tampere: Tampere University Press; 2002. p. 1-82.

17. Volcheck GW. Does rhinitis lead to asthma. Evidence for the oneairway hypothesis. Postgrad med 2004; 115(5):65-8

18. Shusterman D. Review of the upper airway, including olfaction, as mediator of symptoms. Env Health Perspect 2002; 100(S4) 649-53.

19. Doty RL. Intranasal trigeminal chemoreception: anatomy, physiology and psychophysics. In: Doty RL, editor. Handbook of olfaction and gustation. $1^{\text {st }}$ ed. New York: Marcell Dekker. 1995. p. 821-33.

20. Klinghardt DK. The Sphenopalatine ganglion (SPG) and environmental sensitivity. Lecture on 23rd Annual International Symposium on Man and his Environment. June 9-12, 2005. Dallas Texas. Available online at URL http://www.naturaltherapy.com. Accessed March 20, 2006
21. Boyd J. Pathophysiology of migraine and rationale for a targeted approach and prevention. Available online at URL http://www.migraineprevention.com/index/html. Accessed February $15,2006$.

22. Utomo H. Sensitization of the sphenopalatine ganglion by periodontal inflammation: a possible etiology for sinusitis and headache in children. Majalah Kedokteran Gigi FKG Universitas Airlangga. 2006; 39(2):63-7.

23. Grossan M Asthma and sinusitis. Available online at URL http://www. emedicine.com/ent/contents.htm. Accessed in June 6, 2006.

24. Chih-Feng T, Baraniuk JN. Upper airway neurogenic mechanisms. Cur Al Clin Immunol 2002; 2(1):11-9. 